\title{
Helicobacter pylori-induced gastric cancer is orchestrated by MRCK $\beta$-mediated Siah2 phosphorylation
}

Pragyesh Dixit ${ }^{1}$, Shrikant B. Kokate ${ }^{1,6}$, Indrajit Poirah' ${ }^{1}$, Debashish Chakraborty' ${ }^{1}$ Duane T. Smoot ${ }^{2}$, Hassan Ashktorab ${ }^{3}$, Niranjan Rout ${ }^{4}$, Shivaram P. Singh ${ }^{5}$ and Asima Bhattacharyya ${ }^{1 *}$ (D)

\begin{abstract}
Background: Helicobacter pylori-mediated gastric carcinogenesis is initiated by a plethora of signaling events in the infected gastric epithelial cells (GECs). The E3 ubiquitin ligase seven in absentia homolog 2 (Siah2) is induced in GECs in response to H. pylori infection. Posttranslational modifications of Siah2 orchestrate its function as well as stability. The aim of this study was to evaluate Siah2 phosphorylation status under the influence of H. pylori infection and its impact in gastric cancer progression.
\end{abstract}

Methods: H. pylori-infected various GECs, gastric tissues from H. pylori-infected GC patients and H. felis-infected C57BL/6 mice were evaluated for Siah2 phosphorylation by western blotting or immunofluorescence microscopy. Coimmunoprecipitation assay followed by mass spectrometry were performed to identify the kinases interacting with Siah2. Phosphorylation sites of Siah2 were identified by using various plasmid constructs generated by site-directed mutagenesis. Proteasome inhibitor MG132 was used to investigate proteasome degradation events. The importance of Siah2 phosphorylation on tumorigenicity of infected cells were detected by using phosphorylation-null mutant and wild type Siah2 stably-transfected cells followed by clonogenicity assay, cell proliferation assay, anchorage-independent growth and transwell invasion assay.

Results: Siah2 was phosphorylated in H. pylori-infected GECs as well as in metastatic GC tissues at residues serine $^{6}\left(\mathrm{Ser}^{6}\right)$ and threonine ${ }^{279}\left(\mathrm{Thr}^{279}\right)$. Phosphorylation of Siah2 was mediated by MRCK $\beta$, a Ser/Thr protein kinase. MRCK $\beta$ was consistently expressed in uninfected GECs and noncancer gastric tissues but its level decreased in infected GECs as well as in metastatic tissues which had enhanced Siah2 expression. Infected murine gastric tissues showed similar results. MRCK $\beta$ could phosphorylate Siah2 but itself got ubiquitinated from this interaction leading to the proteasomal degradation of MRCK $\beta$ and use of proteasomal inhibitor MG132 could rescue MRCK $\beta$ from Siah2mediated degradation. Ser $^{6}$ and Thr $^{279}$ phosphorylated-Siah2 was more stable and tumorigenic than its non-phosphorylated counterpart as revealed by the proliferation, invasion, migration abilities and anchorage-independent growth of stable-transfected cells.

Conclusions: Increased level of $\mathrm{Ser}^{6}$ and $\mathrm{Th}^{279}$-phosphorylated-Siah2 and downregulated MRCK $\beta$ were prominent histological characteristics of Helicobacter-infected gastric epithelium and metastatic human GC. MRCK $\beta$-dependent

\footnotetext{
*Correspondence: asima@niser.ac.in

${ }^{1}$ School of Biological Sciences, National Institute of Science Education and Research (NISER) Bhubaneswar, HBNI, P.O. Bhimpur-Padanpur, Via Jatni, Khurda 752050, Odisha, India

Full list of author information is available at the end of the article
}

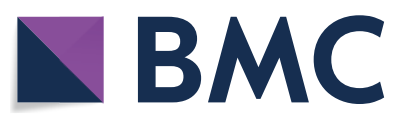

The Author(s) 2021. Open Access This article is licensed under a Creative Commons Attribution 4.0 International License, which permits use, sharing, adaptation, distribution and reproduction in any medium or format, as long as you give appropriate credit to the original author(s) and the source, provide a link to the Creative Commons licence, and indicate if changes were made. The images or other third party material in this article are included in the article's Creative Commons licence, unless indicated otherwise in a credit line to the material. If material is not included in the article's Creative Commons licence and your intended use is not permitted by statutory regulation or exceeds the permitted use, you will need to obtain permission directly from the copyright holder. To view a copy of this licence, visit http://creativecommons.org/licenses/by/4.0/. The Creative Commons Public Domain Dedication waiver (http://creativeco mmons.org/publicdomain/zero/1.0/) applies to the data made available in this article, unless otherwise stated in a credit line to the data. 
Siah2 phosphorylation stabilized Siah2 which promoted anchorage-independent survival and proliferative potential of GECs. Phospho-null mutants of Siah2 (S6A and T279A) showed abated tumorigenicity.

Keywords: Cdc42BPB, E3 ubiquitin ligase, Gastric cancer, Helicobacter pylori, Host-pathogen interaction, Proteasome

\section{Background}

GC progresses through multiple stages and involves various factors. H. pylori (a class I carcinogen) is the prime factor for increased risk of GC. This pathogen infects human stomach and acquires a number of adaptations to survive in its harsh ecological niche, the pylorus part of the human stomach. Infection of $H$. pylori initiates various pathophysiological events in the gastric epithelium which promote GC [1-4].

Siah proteins belonging to the E3 ubiquitin ligase family are associated with several cancers including GC [5-7]. H. pylori infection upregulates Twist-related protein 1 and E26 transformation-specific sequence 2 which transcriptionally increase Siah2 level. Increase in Siah2 aids in H. pylori-mediated GC progression [8]. Siah2 enhances invasiveness and progression of GC through the regulation of degradation of its various binding partners [9]. Siah2 function and subcellular localization are modulated by post-translational modifications. Acetylation of Siah2 promotes Siah2 stabilization in GECs infected with $H$. pylori and induces Siah2-mediated prolyl hydroxylase 3 degradation, thus contributing to infection-induced hypoxia [7]. Siah2 phosphorylation by p38 mitogen-activated protein kinase and dual-specificity tyrosine phosphorylation-regulated 2 kinases play critical roles in hypoxic stress response by regulating prolyl hydroxylase 3 [10, 11]. Phosphorylation of Siah2 by Src kinase transforms breast cancer by degrading CCAAT/ enhancer-binding protein delta, a transcription factor known for its tumor-suppressive functions [12]. p53 kinase homeodomain-interacting protein kinase 2 is also known to phosphorylate Siah2, thus rendering it more active and resulting in its ubiquitination followed by degradation. This further leads to the downregulation of p53 [13]. Serine-threonine checkpoint kinase 2-mediated Siah2 phosphorylation leads to checkpoint kinase 2 downregulation having consequences in DNA damage response and cell cycle control [14]. However, the status and mechanism of Siah2 phosphorylation and its importance in H. pylori-mediated GC remain elusive.

Keeping in purview the crucial role of Siah2 proteins in GC progression, we studied Siah2 phosphorylation status in the $H$. pylori-infected gastric epithelium and investigated the effect of phospho-Siah2 in GC progression. Here we report that $H$. pylori infection enhances Siah2 phosphorylation through myotonic dystrophy kinase-related Cdc42-binding kinase $\beta$ (MRCK $\beta)$, a Ser/
Thr kinase. MRCK $\beta$ remains anchored by a tight junction protein zonula occludens-1 at the leading-edge of the invasive cell and controls migration [15]. Squamous cancer cell migration [16] and ErbB2-driven breast cancer invasion [17] are MRCK $\beta$-mediated. MRCK $\beta$ has also been implicated to play a role in skin cancer progression [18] and HeLa cell elongation [19]. This study identifies that MRCK $\beta$-mediated Siah2 phosphorylation at Ser ${ }^{6}$ and $\mathrm{Thr}^{279}$ increases Siah2 stability and positively contributes in the GC progression. The presence of phosphorylated Siah2 in the human GC biopsy tissues also reveal its potential diagnostic and therapeutic importance.

\section{Methods \\ Cells, Helicobacter strains and reagents}

MKN45 and AGS cells (human gastric epithelial cell lines), cag PAI positive $H$. pylori strain 26695, cag PAI negative $H$. pylori strain $8-1$ and $H$. felis strain 49179 were purchased from the American Type Culture Collection (Manassas, VA). Immortalized non-neoplastic HFE145 GECs, other mentioned cell lines and $H$. pylori were maintained as per established protocols $[7,20]$. Cells were infected with $H$. pylori using various multiplicity of infection (MOI) for the indicated durations. Proteasomal inhibitor MG132 or Z-Leu-Leu-Leu-al (Millipore-Sigma, St. Louis, MO) at $50 \mu \mathrm{M}$ concentration was used in assays aiming to identify involvement of proteasomal degradation. Gentamicin (Himedia, Nashik, India) was used in clonogenicity assay to kill extracellular $H$. pylori at $150 \mu \mathrm{g} / \mathrm{ml}$ concentration for $3 \mathrm{~h}$.

\section{Expression plasmids and site-directed mutagenesis}

Full-length human siah2 (Origene Technologies, MD; hereafter referred to as siah2 WT) and eukaryotic expression vector pcDNA3.1+(Thermo Fisher Scientific, MA) were purchased. Various siah2 mutants were generated from the siah2 WT constructs by site-directed mutagenesis (QuikChange site-directed mutagenesis kit, Agilent Technologies, CA) using suggested procedure. Primers used were as follows:

For siah2 S6A: 5' CATGAGCCGCCCGTCCGCCAC CGGCCCCAGCGC 3'.

For siah2 T275A: 5' GGGAACCGGCGGAGATTG GCCTGGGAGGCCACGCCCC 3'.

For siah2 T279A: 5' AGATTGACCTGGGAGGCC GCGCCCCGTTCGATTCATG 3. 
For siah2 S282A: 5' TGGGAGGCCACGCCCCGT GCGATTCATGACGGTGTGG 3.'

pEGFP-N1-Cdc42BPB $(m r c k \beta)$ was gifted by Prof. Naoki Mochizuki, National Cerebral and Cardiovascular Center, Osaka, Japan.

\section{Transient transfection and generation of stable cell lines}

$24 \mathrm{~h}$ post-plating and $1 \mathrm{~h}$ prior to transfection, spent media was discarded and replenished by $10 \%$ FBS (HiMedia) containing RPMI1640 media (HiMedia). Cells were transfected with DNA, P3000 reagent and Lipofectamine 3000 (Invitrogen, CA) as per the company's instructions. When required, cells were transfected with control siRNA (Santa Cruz Biotechnology, TX) and $m r c k \beta$ siRNA (Santa Cruz Biotechnology) using Lipofectamine 3000. Similar protocol was used for transfection of control siRNA (Origene) and siRNA of siah2 (Origene). G418 solution (Millipore-Sigma) was used to select stablytransfected clones following standard protocol.

\section{Western blotting and coimmunoprecipitation assays}

Western blotting was performed following standard protocols $[7,9,20]$. Briefly, total cell lysates were prepared from GECs (with or without H. pylori infection), run on SDS-PAGE and electro-transferred onto PVDF membrane. MRCK $\beta$ and Siah2 (Santa Cruz Biotechnology) Phospho-Serine (Millipore-Sigma), Phospho-Threonine (Cell Signaling Technology, MA), Ubiquitin (Cell Signaling Technology) and GAPDH (Abgenex, Bhubaneswar, India) primary antibodies were used to probe electroblotted membranes. Customized human P-Ser ${ }^{6}-\mathrm{Siah} 2$ and $\mathrm{P}-\mathrm{Thr}^{279}$-Siah2 antibodies were generated (Bioklone Biotech Pvt. Ltd., Chennai, India). Immunoblots were detected by Thermo Scientific SuperSignal West Femto Chemiluminescent Substrate kit (Thermo Fisher Scientific, IL) and images were captured using BioRad Chemidoc XRS+. Densitometric analysis was done using ImageLab software (Bio-Rad, CA). Whole-cell lysates were immunoprecipitated using Siah2 antibody and immunoprecipitates were detected by western blotting.

\section{Immunofluorescence microscopy and image analysis}

GECs were cultured on coverslips, infected with $H$. pylori (MOI 200, $12 \mathrm{~h}$ ) and processed for immunofluorescence microscopy. Cells were incubated with desired specific antibodies at $4{ }^{\circ} \mathrm{C}$ for overnight using manufacturer's recommended dilution. Fluorophore Alexa Fluor-488 and 594-tagged secondary antibodies (Invitrogen, OR) were used. 4', 6-Diamidino-2-phenylindoledihydrochloride (DAPI; Invitrogen) was used for $20 \mathrm{~min}$ to stain nuclei. Digital images were taken by Nikon microscope (Eclipse Ti-U, Nikon, Tokyo, Japan) fitted with the digital monochrome camera DS Qi2 (Nikon).
Confocal images were captured on Leica DMi8 confocal microscope (Leica, Germany) using Leica Application Suite X software. Captured images were processed and analysed using NIS Advanced Research software (Nikon).

\section{Human gastric biopsy and mouse gastric tissue collection} Metastatic stage IV antral gastric adenocarcinoma biopsy samples were obtained from the gastric antrum of urease-test positive GC patients $(n=9)$. A noncancerous biopsy sample from the adjacent area was also obtained from each patient which was used as a paired-control. A prior-informed signed consent was obtained for all patients and patient privacy was preserved. Patients who were treated for $H$. pylori eradication were not included in the study. The protocol used for biopsy collection was approved by the Institutional Ethics Committee for Human Research, National Institute of Science Education and Research and was in compliance with the Helsinki Declaration (2013), World Medical Association.

7-8 weeks old C57BL/6 mice (procured from National Centre for Laboratory Animal Sciences of the National Institute of Nutrition, Hyderabad, India) were blindly divided into uninfected $(\mathrm{n}=16)$ and infected $(\mathrm{n}=16)$ irrespective of their sex and were infected with $H$. felis or $1 \mathrm{X}$ PBS following a standard protocol [21]. After 18 months of infection, mice were euthanized and their stomachs were collected. This study was approved by the Institutional Animal Ethics Committee of National Institute of Science Education and Research. Samples were fixed using 4\% paraformaldehyde and sectioned at $5 \mu \mathrm{m}$ thickness using a cryomicrotome.

\section{MTT assay}

Proliferation of pcDNA3.1+, siah2 WT or siah2 phospho-null mutant (siah2 S6A and siah2 T279A) stabletransfected MKN45 cells infected with $200 \mathrm{MOI} H$. pylori for $12 \mathrm{~h}$ was assessed using a MTT assay kit (EZcount MTT cell assay kit, Himedia) following the company's recommended protocol.

\section{Soft agar assay}

Stably-transfected MKN45 cells were used to assess anchorage-independent growth following our previouslyestablished protocols $[8,9]$.

\section{Clonogenic survival assay}

pcDNA3.1+, WT, S6A and T279A siah2-expressing MKN45 stable cells were seeded in 24-well cell culture dishes with a density of $16.6 \times 10^{4}$ followed by infection for $12 \mathrm{~h}$ at 200 MOI. Next, extracellular pathogens were killed by using $150 \mu \mathrm{g} / \mathrm{ml}$ gentamicin for $3 \mathrm{~h}$. Cells were trypsinized followed by seeding in $60 \mathrm{~mm}^{2}$ dishes with 1000 cells/dish. After 3 weeks of incubation, cells were 


\section{(See figure on next page.)}

Fig. 1 Siah2 phosphorylation is increased in H. pylori-infected GEC. a Table showing partial or full Siah degron motifs in the protein sequence of kinases identified by mass spectrometry. $\mathbf{b}$ Western blotting of total cell extracts prepared from the uninfected and H. pylori-infected (200 MOI) MKN45 cells at 6, 12, and 24 h post-infection show increased P-Ser-Siah2, P-Thr-Siah2 and Siah2 levels. Histograms clearly represent a significant increment of P-Ser-Siah2, P-Thr-Siah2 and Siah2 at all time points. Statistical significance is determined by two-way ANOVA followed by Tukey's post hoc analysis. c Western blot of whole cell lysate from MKN45 cells uninfected and infected with H. pylori (MOl 100, 200 and 300 MOI for 12 h). Graphical representation reveals a significant increase of P-Ser-Siah2, P-Thr-Siah2 and Siah2 with $200 \mathrm{MOl}$ of infection. One-way ANOVA is used to determine statistical significance. $\mathbf{d}$ A representative western blot from uninfected and infected (200 MOl for $12 \mathrm{~h}$ of H. pylori 26695 strain [cag PAl +] or 8-1 strain [cag PAI - ]) MKN45 cells representing P-Ser-Siah2, P-Thr-Siah2 and Siah2. GAPDH is used as a loading control. All graphical data are mean $\pm \operatorname{sem}(n=3) .{ }^{*} P<0.05,{ }^{* *} P<0.01,{ }^{* * *} P<0.001,{ }^{* * * *} P<0.0001$

fixed with $4 \%$ paraformaldehyde. After fixing and staining with crystal violet $(0.5 \%)$, the number of colonies formed were counted.

\section{In vitro invasion assay}

pcDNA3.1+, WT, S6A and T279A siah2-expressing AGS stable cells were seeded on cell culture inserts with 0.3 $\mathrm{cm}^{2}$ polyethylene terephthalate membrane $(8-\mu \mathrm{m}$ pore size and 6.4-mm diameter; Falcon Corning, MA) coated with growth factor-reduced standard Matrigel matrix (Becton Dickinson, NJ) and further processed according to our previously reported protocol $[9,22]$. Images were captured from at least three different fields using an inverted microscope as already mentioned. Invaded cells were counted.

\section{Cell population doubling assay}

pcDNA3.1+, WT, S6A and T279A siah2-expressing MKN45 stable cells were seeded in 24 well plate at a density of $16.6 \times 10^{4}$ cells/well. After $24 \mathrm{~h}$ of incubation, cells were infected with $200 \mathrm{MOI}$ of $\mathrm{H}$. pylori for $12 \mathrm{~h} .150 \mu \mathrm{g} /$ $\mathrm{ml}$ gentamicin was used for $3 \mathrm{~h}$ to kill extracellular bacteria. Next, cells were trypsinized and 10,000 cells were seeded in 24 well plate. After seeding, the number of cells were counted on day 2, 3 and 4 . The population doubling (PD) was calculated using the formula below [23, 24] where Ns and Nh are the number of seeded and harvested cells, respectively:

$$
\mathrm{PD}=[\log (\mathrm{Nh})-\log (\mathrm{Ns})] / \log 2
$$

The cumulative population doubling (cPD) level was calculated by summation of the PD values obtained for each day and represented as graphs with cPD at y-axis [25].

\section{Statistical analysis}

GraphPad Prism 7.00 software (GraphPad, CA) was used for statistical analyses. Values represent mean \pm sem. $P<0.05$ determined statistical significance using either one-way or two-way ANOVA followed by Tukey's post hoc test. All results represent at least three independent experimental repeats.

\section{Results}

H. pylori infection enhances Siah2 phosphorylation

Our group identified that Siah2 was optimally induced by $H$. pylori $12 \mathrm{~h}$ after infection and at $200 \mathrm{MOI}[7,9]$. In order to identify kinases that interact with Siah2 and to enrich interaction signals, overexpression-immunoprecipitation assay was performed. MKN45 cells were transfected with either siah2 overexpression plasmid or the vector control followed by H. pylori 26695 (cag $\mathrm{PAI}+$ strain) infection for $12 \mathrm{~h}$ at $200 \mathrm{MOI}$. Whole cell lysates were subjected to immunoprecipitation with Siah2 antibody and immunocomplexes were separated by SDS-PAGE followed by staining of the gel with Coomassie brilliant blue (R-250). Bands were cut and mass spectrometry was done. Among the identified kinases from the Siah2-immunocomplex was MRCK $\beta$, a Ser/ Thr kinase and two isoforms of 6-phosphofructokinase (6-PFK). Our analysis further revealed that MRCK $\beta$ has four partial and one full Siah-degron motifs but 6-PFK isoforms have only one partial degron motif (Fig. 1a). The presence of Siah-degron motifs indicate towards the likelihood of a molecule to be a Siah-substrate [23, 24]. Therefore, our observation pointed to the potential of MRCK $\beta$ being a Siah2-substrate. LR_PPI prediction [26] of Siah2-MRCK $\beta$ interaction also suggested of high probability (0.9943) of their interaction (Additional file 1: Fig. S1).

In order to assess Siah2 phosphorylation after $H$. pylori infection, MKN45 cells were challenged with 200 MOI of H. pylori 26695 for various durations. From the western blot results, enhanced Siah2 level as well as increase in P-Ser-Siah2 and P-Thr-Siah2 were noticed in H. pyloriinfected cells (Fig. 1b), reaching optimal levels at $12 \mathrm{~h}$ post infection. Siah2 phosphorylation was optimal at $12 \mathrm{~h}$ of $H$. pylori infection at 200 MOI (Fig. 1c). To assess cag PAI-dependence on Siah2 Ser/Thr phosphorylation, we infected MKN45 cells with MOI 200 of the cag PAI $+H$. pylori strain 26695 or the cag PAI - H. pylori strain 8-1 
a

\begin{tabular}{|c|c|c|c|}
\hline Kinase & $\begin{array}{l}\text { Protein } \\
\text { Accession No. }\end{array}$ & Score & $\begin{array}{l}\text { Siah Degron Motif } \\
\text { (amino acid residues) }\end{array}$ \\
\hline $\begin{array}{l}\text { 6-phosphofructokinase, platelet } \\
\text { type }\end{array}$ & Q01813 & 67.68 & $\begin{array}{l}\text { Partiall Core: } \\
\text { VLP (482-484) }\end{array}$ \\
\hline $\begin{array}{l}\text { Serine/threonine-protein kinase } \\
\text { MRCK } \beta\end{array}$ & Q9Y5S2 & 61.32 & $\begin{array}{l}\text { Partial/ Core: } \\
\text { VCP (1074-1076), } \\
\text { VAP (1371-1373), } \\
\text { VNP (1409-1411), } \\
\text { VDP (1448-1450) } \\
\text { Full: PLSAVPP(1608-1614) }\end{array}$ \\
\hline $\begin{array}{l}\text { 6-phosphofructokinase, liver } \\
\text { type }\end{array}$ & P17858 & 36.33 & $\begin{array}{l}\text { Partiall Core: } \\
\text { VIP (523-525) }\end{array}$ \\
\hline
\end{tabular}

b

\begin{tabular}{|c|c|c|}
\hline \\
\hline \multicolumn{3}{|c|}{$35 \mathrm{kDa} \underset{\square \text { Uninfected } H \text {. pylori }}{\text { P-Th }}$} \\
\hline $35 \mathrm{kDa}$ & $4+1+2$ & P-Ser-Siah2 \\
\hline $35 \mathrm{kDa}$ & $\sqrt{25+408}$ & Siah2 \\
\hline $37 \mathrm{kDa}$ & ---1 & GAPDH \\
\hline
\end{tabular}

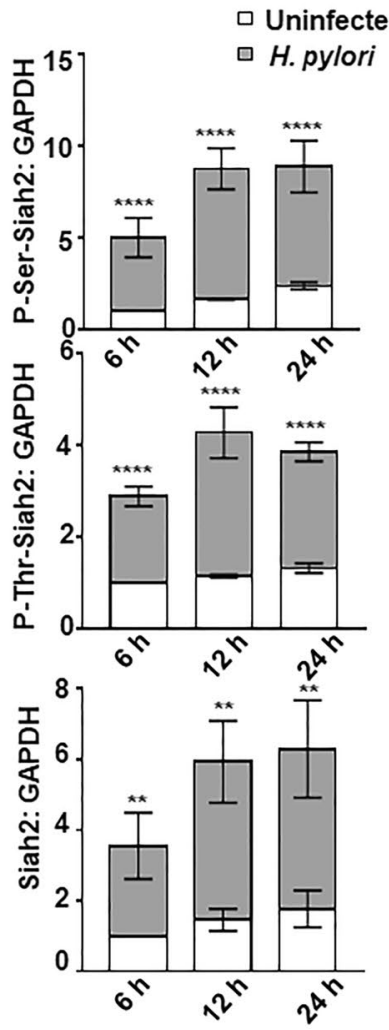

C
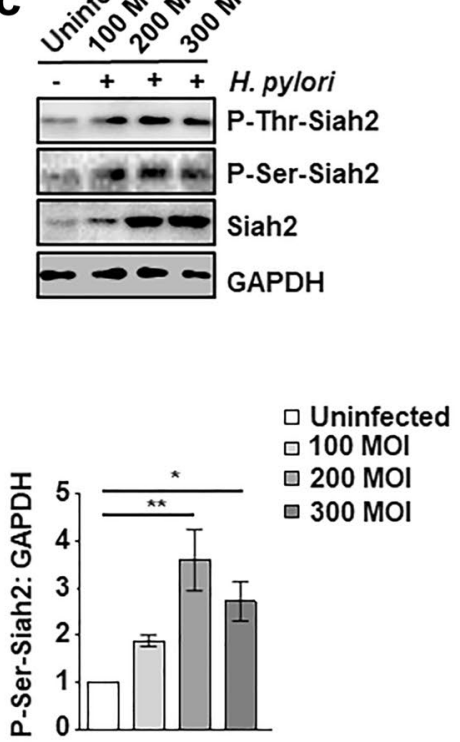

I $4_{1} \stackrel{*}{-T}$ d

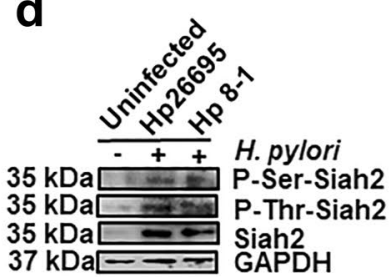

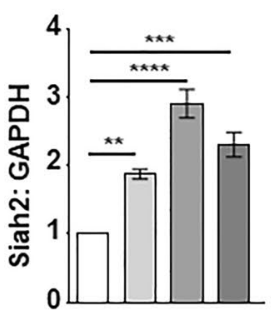




\begin{abstract}
(See figure on next page.)
Fig. 2 MRCK $\beta$ is decreased in H. pylori-mediated GC and interacts with Siah2. a Western blot of whole cell extracts from uninfected and $H$. pylori-infected MKN45 cells (at 6, 12, and 24 h of infection with $200 \mathrm{MOl}$ of H. pylori 26695) showing protein level of MRCKR. GAPDH is a loading control. Bar graph clearly indicates a time-dependent decrease of MRCK $\beta$ after H. pylori infection. Two-way ANOVA followed by Tukey's post hoc analysis is used to determine statistical significance. b Western blotting of total cell lysate from uninfected and H. pylori-infected (at MOI 100, 200 and 300) MKN45 cells showing MRCK $\beta$ protein. GAPDH is used as a loading control. Graph represents decrease of MRCK $\beta$ protein as compared to uninfected control. One-way ANOVA is used to determine statistical significance. c A representative western blot of infected $(200 \mathrm{MOI}$ for $12 \mathrm{~h}$ of $\mathrm{H}$. pylori 26695 or 8-1 strain) or uninfected MKN45 cells showing MRCK $\beta$ protein level $(n=3)$. GAPDH is used as a loading control. $\mathbf{d}$ A representative $(n=9)$ immunofluorescence micrograph of human metastatic GC biopsy tissue samples showing the status of MRCK $\beta$. Tissues are sectioned at $5 \mu \mathrm{m}$ thickness. Images are captured using 40X objective and scale bars $=50 \mu \mathrm{m}$. e Western blot of whole cell lysate from uninfected and infected (H. pylori at $200 \mathrm{MOI}$ for $12 \mathrm{~h}$ ) MKN45 cells subjected to co-immunoprecipitation using Siah2 antibody reveals that Siah2 interacts with MRCK 3 . IgG band is used to indicate equal loading. $\mathbf{f} A$ representative western analysis indicating ubiquitination status of MRCK $\beta$ from uninfected or infected (H. pylori at $200 \mathrm{MOI}$ for $12 \mathrm{~h}$ ) and 50 MM MG132-treated MKN45 cells. Reprobed bands of MRCK 3 , Siah2 and GAPDH. g Western blotting of total cell lysates from uninfected or infected ( $\mathrm{H}$. pylori at $200 \mathrm{MOI}$ for $12 \mathrm{~h}$ ) and $50 \mu \mathrm{M}$ MG132-treated MKN45 cells subjected to co-immunoprecipitation using Siah2 antibody showing MRCK $\beta$ and Siah2 protein status. IgG band is used as the indicator of equal loading. All data are mean \pm sem ( $n=3$ ). ${ }^{* *} P<0.01,{ }^{* * *} P<0.001$
\end{abstract}

for $12 \mathrm{~h}$. Siah2 phosphorylation was induced by both the strains as detected by western blot (Fig. 1d).

MRCK $\beta$ protein is downregulated in $H$. pylori-infected GECs Several kinases interacting with Siah2 get degraded by phosphorylation-mediated augmented Siah2 activity $[11,13,14]$. Necessitated by the presence of Siah-degron motifs in the MRCK $\beta$ protein sequence, examining $H$. pylori-driven regulation of the latter was imperative. The GEC MKN45 was therefore, challenged with H. pylori (MOI 200) for various time periods. Western blot results revealed that MRCK $\beta$ was significantly low in the infected cells at $12 \mathrm{~h}$ post infection as compared to the uninfected lanes (Fig. 2a). We also compared 100, 200 and 300 MOIs to find the optimal infection ratio to downregulate MRCK $\beta$. Interestingly, at $200 \mathrm{MOI}$ and $12 \mathrm{~h}$ of infection, MRCK $\beta$ protein level was optimally decreased in MKN45 cells as compared to the uninfected cells (Fig. 2b). In order to identify the role of cag PAI on MRCK $\beta$ decrease, MKN45 cells were infected with 200 MOI of cag PAI $+H$. pylori strain 26695 and cag PAI - H. pylori strain 8-1 at 200 MOI. Analysis of western blot showed that MRCK $\beta$ downregulation was cag PAI-independent (Fig. 2c). To study the MRCK $\beta$ status in human metastatic GC, antral biopsy tissues were obtained from consenting individuals followed by immuno-staining with MRCK $\beta$ antibody. A profound decrease in MRCK $\beta$ protein was observed in metastatic samples as compared to their paired-controls (Fig. 2d).

MRCK $\beta$ interaction with Siah2 was confirmed by infecting MKN45 cells with $H$. pylori for $12 \mathrm{~h}$ at 200 MOI followed by co-immunoprecipitation using Siah2 antibody. Western blotting of the immunocomplexes confirmed of MRCK $\beta$-Siah2 interaction and indeed, decreased MRCK $\beta$ interaction was observed in $H$. pylori-infected cells (Fig. 2e). Siah2 ubiquitinates most of its interacting partners and marks them for proteasome-mediated degradation [27, 28]. To assess the ubiquitination status of MRCK $\beta$ in infected cells, MKN45 cells were treated with $50 \mu \mathrm{M}$ MG132, a proteasomal inhibitor, along with $H$. pylori infection for $12 \mathrm{~h}$ at 200 MOI. Western blotted membranes were probed with ubiquitin antibody to detect formed ubiquitin aggregates. MG132 treatment rescued ubiquitinated proteins in the infected cells (Fig. 2f). Reprobing of the band for MRCK $\beta$ confirmed about the ubiquitination of the protein in the infected GECs. Further, we wanted to assess the interaction status of MRCK $\beta$-Siah2 after MG132 treatment. For this, MKN45 cells were treated with $50 \mu \mathrm{M}$ of MG132 followed by $H$. pylori infection for $12 \mathrm{~h}$ or were left untreated. Immunoprecipitation of whole cell lysates were performed using Siah2 antibody and the western blotting revealed less MRCK $\beta$ decrease in MG132treated infected cells (Fig. 2g).

\section{Siah2 degrades MRCK $\beta$ in H. pylori-infected GECs}

To investigate whether MRCK $\beta$ decrease was correlated with Siah2 increase, we infected pcDNA3.1+ and siah2 WT MKN45 stable cells with $H$. pylori. Immunofluorescence microscopy revealed that MRCK $\beta$ was downregulated in $H$. pylori-infected cells and this was further decreased in siah2 WT stable cells (Fig. 3a). In order to confirm Siah2-dependent MRCK $\beta$ protein decrease, we transfected MKN45 cells with siRNA of siah2 for $36 \mathrm{~h}$ and infected with $H$. pylori for $12 \mathrm{~h}$. Western blotting revealed that MRCK $\beta$ band was rescued after suppression of siah2, thus confirming about the role of Siah2 in the downregulation of MRCK $\beta$ (Fig. 3b).

\section{Siah2 is phosphorylated and stabilized by MRCK $\beta$}

To study the colocalization of MRCK $\beta$ with that of Siah2, we infected AGS cells with $H$. pylori and cells were processed for immunostaining of Siah2 and MRCK $\beta$. Confocal microscopy showed that Siah2 level was increased 
a

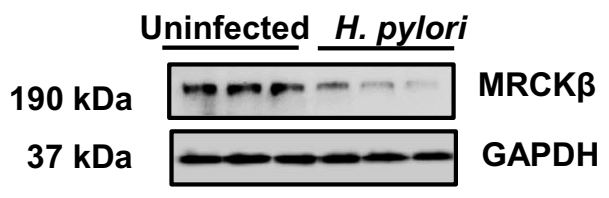

$6 n_{12} n_{2} n_{6} n_{12} n_{24} n$

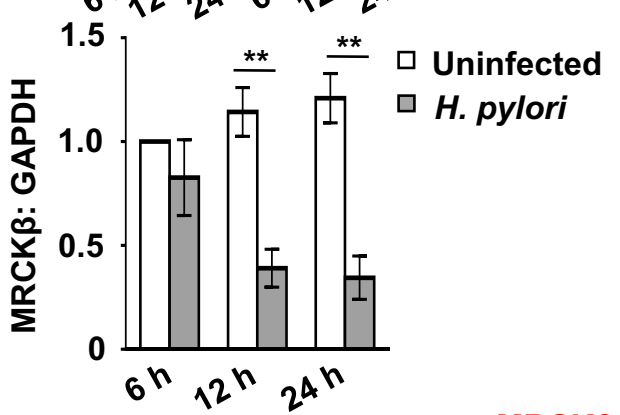

C

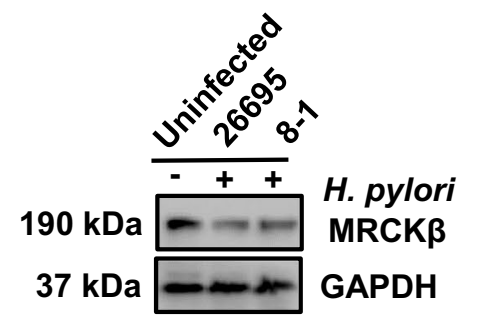

d $\quad$ MRCK $\beta$
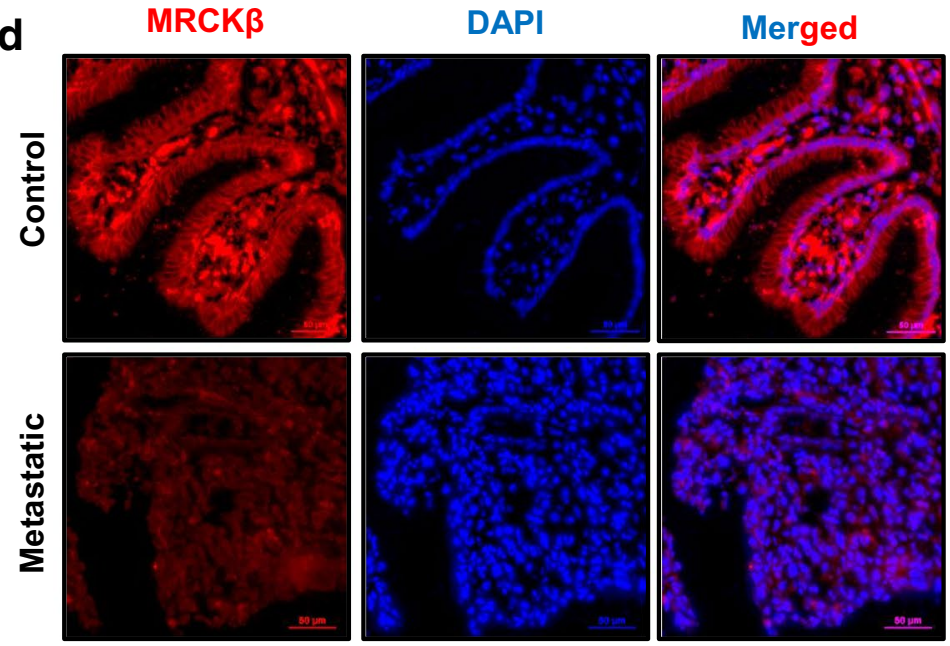

$\mathbf{e}$

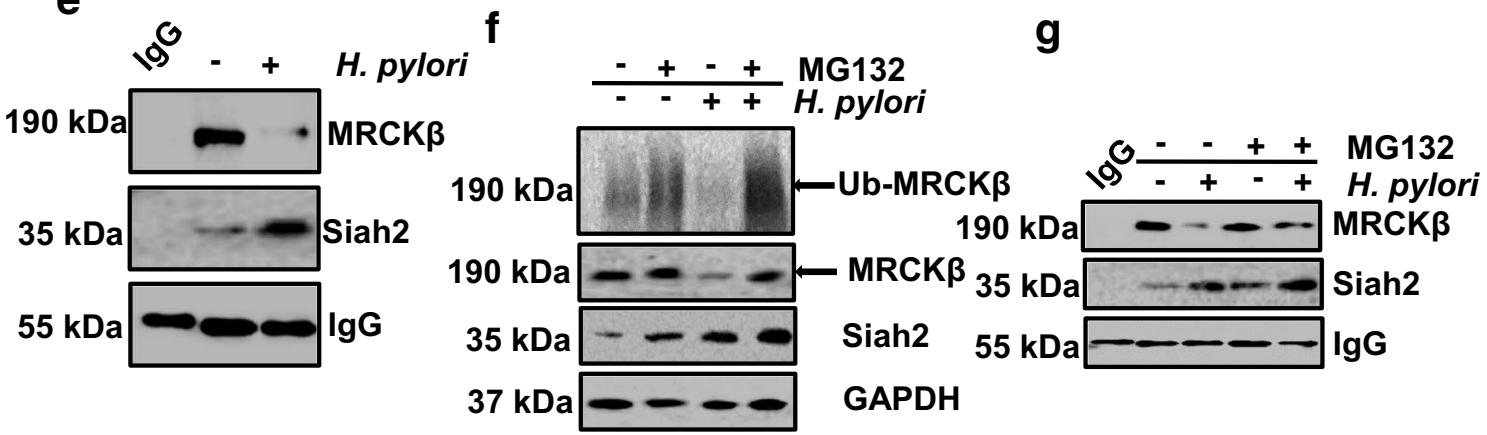

(See figure on next page.)

Fig. 3 MRCK $\beta$ is degraded by Siah2. a Representative $(n=3)$ fluorescence microscopy of pcDNA3.1+ and siah2 WT stably-expressing MKN45 cells infected with H. pylori (200 MOI for $12 \mathrm{~h}$ ). Images are captured using 60X objective and scale bars represent $20 \mu \mathrm{m}$. Bar graphs represent mean fluorescence intensity of Siah2 and MRCK $\beta$ obtained from cells of three independent experiments. Two-way ANOVA is used to determine statistical significance. All data are mean $\pm \operatorname{sem}(n=3) .{ }^{* *} P<0.01,{ }^{* * *} P<0.001,{ }^{* * *} P<0.0001$. b A representative western blot of control siRNA and siah2 siRNA-transfected MKN45 cells followed by $12 \mathrm{~h} \mathrm{H}$. pylori infection showing MRCK $\beta$ and Siah2 protein levels. GAPDH is used as a loading control 


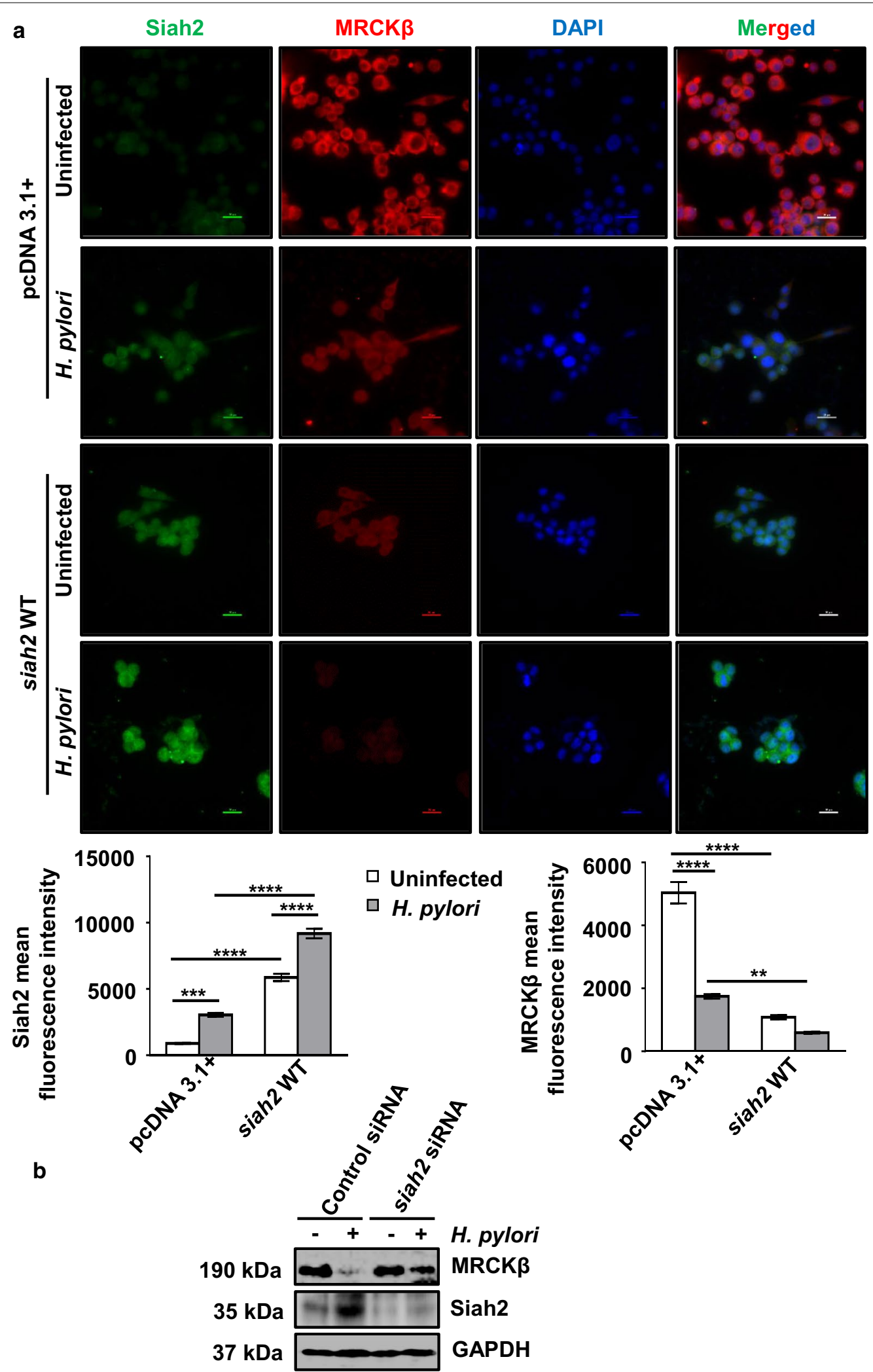




\begin{abstract}
(See figure on next page.)
Fig. 4 MRCK $\beta$ potentiates Siah2 phosphorylation in GECs after H. pylori infection. a Confocal images $(n=3)$ of uninfected and $H$. pylori-challenged (200 MOI for 12 h) AGS cells representing subcellular localization of Siah2 and MRCK $\beta$ proteins. Individual DAPI-stained panels showing nuclear staining are not shown due to constraint of space but the merged panels include DAPI. Scale bar represents $5 \mu$ m. Scatter plots generated by using NIS AR software represent co-localization of MRCK $\beta$ and Siah2 along with their Pearson's correlation (R) and Mander's overlap (MO) values. b A representative $(n=3)$ western blot of whole cell lysates of MKN45 cells transfected with pcDNA3.1+ and mrck $\beta$ and infected with $H$. pylori showing MRCK $\beta$, P-Ser-Siah2, P-Thr-Siah2 and Siah2 protein status. GAPDH = loading control. Bar graphs indicate increase of P-Ser-Siah2, P-Thr-Siah2 and Siah2 after mrck $\beta$ overexpression followed by $12 \mathrm{~h}$ of $\mathrm{H}$. pylori infection. Two-way ANOVA followed by Tukey's post hoc analysis are performed to evaluate statistical significance. Data are mean $\pm \operatorname{sem}(n=3)$. ${ }^{*} P<0.01,{ }^{* * *} P<0.001,{ }^{* * *} P<0.0001$. c Representative western blot $(n=3)$ of pcDNA3.1+, siah2 WT and Siah2 phospho-null mutant-expressing MKN45 stable cells transfected with mrckß followed by H. pylori infection showing P-Ser-Siah2 and Siah2 (above) and P-Thr-Siah2 and Siah2 (below). GAPDH is the loading control. $\mathbf{d}$ Representative western blot $(\mathrm{n}=3$ ) from whole cell lysate of MKN45 cells transfected with either control or mrck $\beta$ siRNA followed by $H$. pylori infection indicating MRCK $\beta$, P-Ser ${ }^{6}$-Siah2, P-Thr ${ }^{279}$-Siah2 and Siah2 proteins. GAPDH is kept as a loading control
\end{abstract}

with a concomitant decrease of MRCK $\beta$. Analysis of the merged image indicated the degree of colocalization between Siah2 and MRCK $\beta$ (Fig. 4a).

To identify the role of MRCK $\beta$ in Siah2 phosphorylation in MKN45 cells, cells were transfected with either the empty vector (pEGFP-N1) or WT $m r c k \beta$ for $36 \mathrm{~h}$ followed by $H$. pylori infection for $6 \mathrm{~h}$ or $12 \mathrm{~h}$. Western blotting and graphical presentation with statistical analysis confirmed that P-Ser-Siah2, P-Thr-Siah2 increased significantly along with Siah2 after $m r c k \beta$ overexpression and $12 \mathrm{~h} \mathrm{H}$. pylori-infection (Fig. 4b), but not at $6 \mathrm{~h}$, when Siah2 did not yet reach its optimal level. In order to identify the probable Ser/Thr phosphorylation residues of Siah2, NetPhos3.0 software [29] was used. Among the predicted Ser/Thr residues of Siah2 with high probability of phosphorylation are shown in Additional file 1: Fig. S2A. Out of the probable phosphorylation residues, phospho-Ser ${ }^{6}$-Siah2 was detected in HeLa cell phosphoproteome [30] but its significance has never been studied. Similarly, the effect of Siah2 phosphorylation at $\mathrm{Thr}^{275}$, $\mathrm{Thr}^{279}$ and $\mathrm{Ser}^{282}$ are also not known. The schematic depiction of the domain arrangement of Siah2 indicates the position of these residues (Additional file 1: Fig. S2B).

In order to study the impact of phosphorylation at these residues, we generated phosphorylation-null mutants of Siah2 (S6A, T275A, T279A and S282A) by site-directed mutagenesis. To consistently study phosphorylation events, we generated pcDNA3.1+, WT Siah2 and Siah2 S6A, T275A, T279A and S282A phospho-null mutant stably-expressing MKN45 cells. In order to identify the phosphorylated residues of Siah2, MKN45 pcDNA3.1+, siah2 WT, Siah2 phospho-null mutant-expressing stable cells were transfected with $m r c k \beta$ and were infected with $H$. pylori or were left uninfected. Siah2 phosphorylation was increased in all $H$. pylori-infected cells except for S6A as detected by western blotting. In the case of phosphonull mutant T279A-expressing cells, the increase in phosphorylation post-infection was lesser than the WT and T275A-infected cells indicating that $\mathrm{Thr}^{279}$ was the prime site for Siah2 phosphorylation in infected GECs (Fig. 4c). The representative data $(\mathrm{n}=3)$ also suggested that Siah2 phosphorylation at $\mathrm{Ser}^{6}$ and $\mathrm{Thr}^{279}$ has role in Siah2 stability. To understand the role of $m r c k \beta$ suppression on Siah2 stability and phosphorylation, $m r c k \beta$ siRNA or control siRNA were transfected in MKN45 cells for $36 \mathrm{~h}$ and were challenged with $H$. pylori for $12 \mathrm{~h}$. Western blot analysis using custom-prepared phospho-specific Siah2 antibodies (P-Ser ${ }^{6}-\mathrm{Siah} 2$ and P-Thr ${ }^{279}$-Siah2) showed abrogation of Siah2 phosphorylation as well as Siah2 downregulation by $m r c k \beta$ suppression (Fig. 4d). These results indicated that MRCK $\beta$-mediated Siah2 phosphorylation at $\mathrm{Ser}^{6}$ and $\mathrm{Thr}^{279}$ contributed in Siah2 stability.

\section{H. pylori infection remarkably enhances Siah2 phosphorylation at Ser ${ }^{6}$ and $\mathrm{Thr}^{279}$ in GECs and in metastatic $\mathrm{GC}$ biopsies}

To assess the status of $\mathrm{Ser}^{6}$ and $\mathrm{Thr}^{279}$-phosphorylated Siah2 in GECs, AGS and MKN45 cells were infected with $H$. pylori for $12 \mathrm{~h}$ and western blotting was performed. Results showed enhanced level of Ser ${ }^{6}$ and $\mathrm{Thr}^{279}$ phosphorylated Siah2 in infected MKN45 as well as AGS cells (Fig. 5a). Response of the immortalized but non-neoplastic GEC HFE145 to $H$. pylori infection was also investigated. For this, HFE145 cells were infected with $H$. pylori for $12 \mathrm{~h}$ and western blotting was performed. Enhanced level of Ser $^{6}$ and $\mathrm{Thr}^{279}$-phosphorylated Siah2 protein and decreased level of MRCK $\beta$ protein were noted in $H$. pylori-infected HFE145 cells (Fig. 5b). Immunofluorescence microscopy of human metastatic GC biopsy samples showed profound increase of Siah2, P-Ser ${ }^{6}$-Siah2 and P-Thr ${ }^{279}$-Siah2 but decreased MRCK $\beta$ in metastatic GC samples as compared to their paired normal gastric tissues (Fig. $5 \mathrm{c}$ and Additional file 1: Fig. S3i-iii). To identify the impact of MRCK $\beta$ on Siah2 phosphorylation at $\mathrm{Ser}^{6}$ and $\mathrm{Thr}^{279}$, immunofluorescence microscopy was 

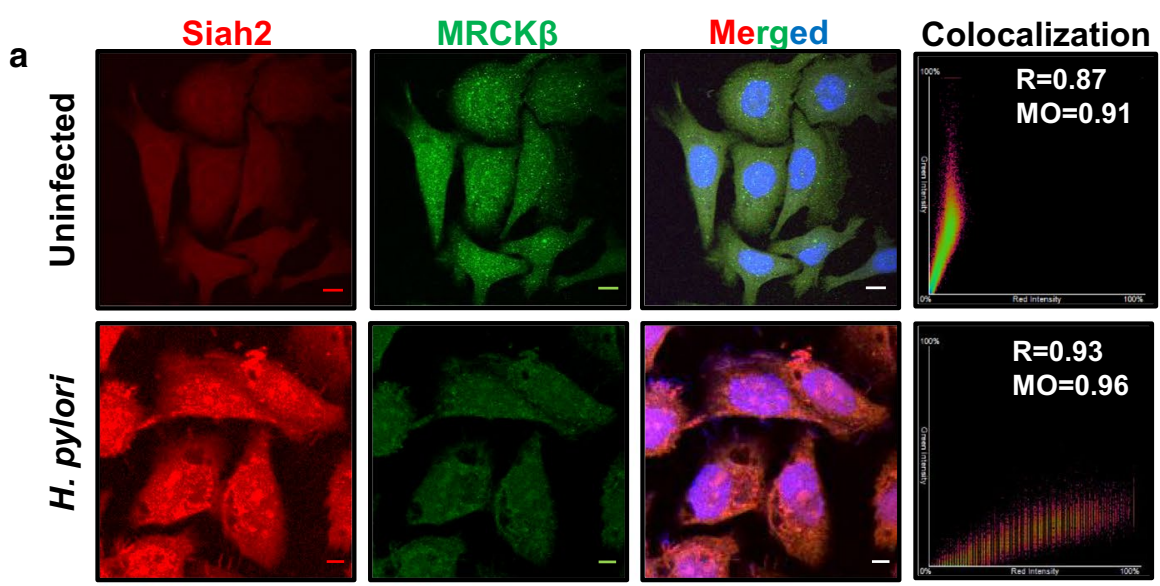

b

b $\quad \frac{\text { Emp vec }}{6 \mathrm{~h} 12 \mathrm{~h}} \frac{m r c k \beta}{6 \mathrm{~h} 12 \mathrm{~h}}$

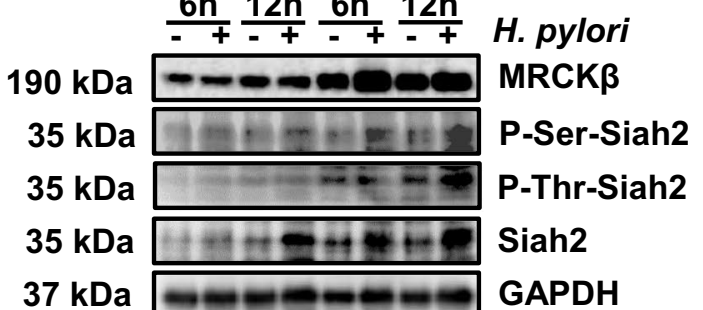

$37 \mathrm{kDa}$

GAPDH
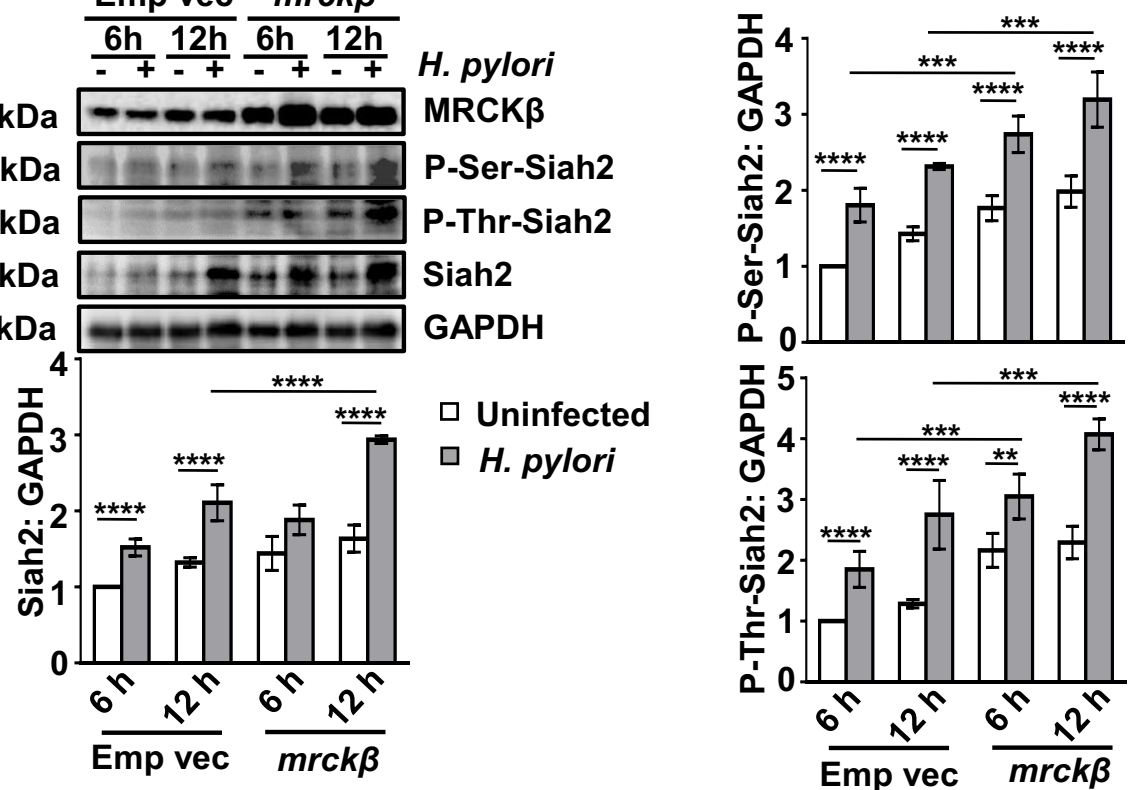

$\square$ Uninfected

$\square$ H. pylori

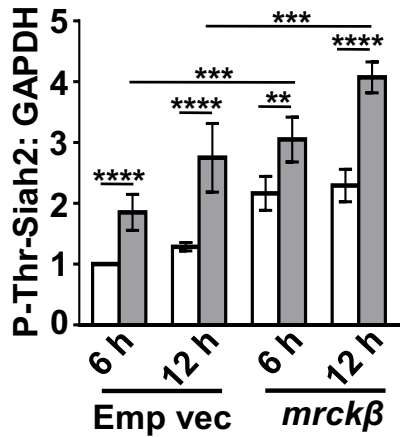

c

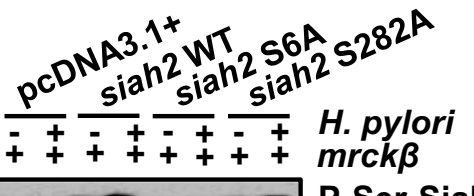

$35 \mathrm{kDa} \longrightarrow$ P-Ser-Siah2

$35 \mathrm{kDa}$

Siah2

$37 \mathrm{kDa}$

GAPDH

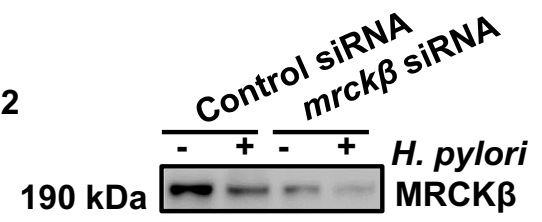

d

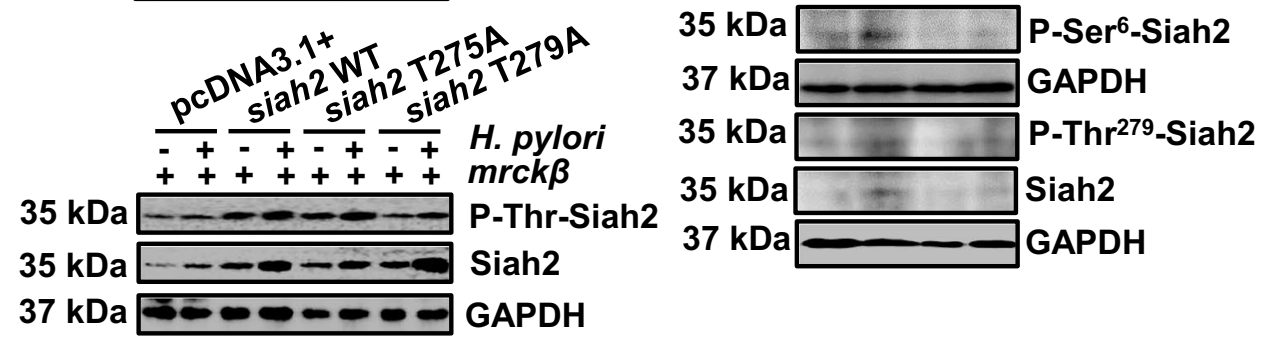


MKN45 AGS

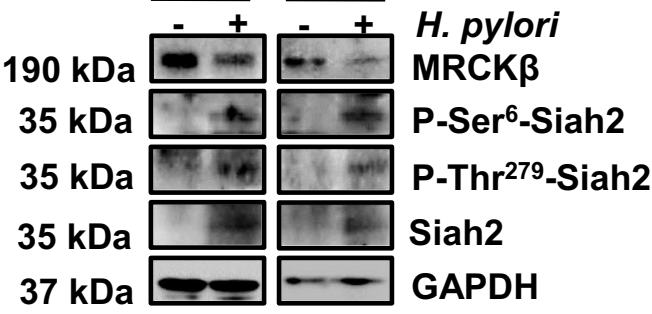

b

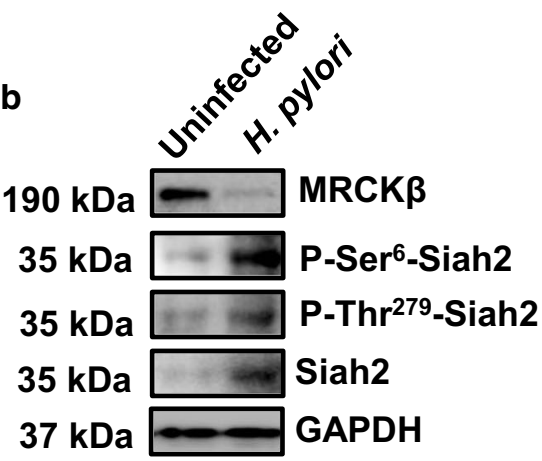

C

P-Ser6_Siah2
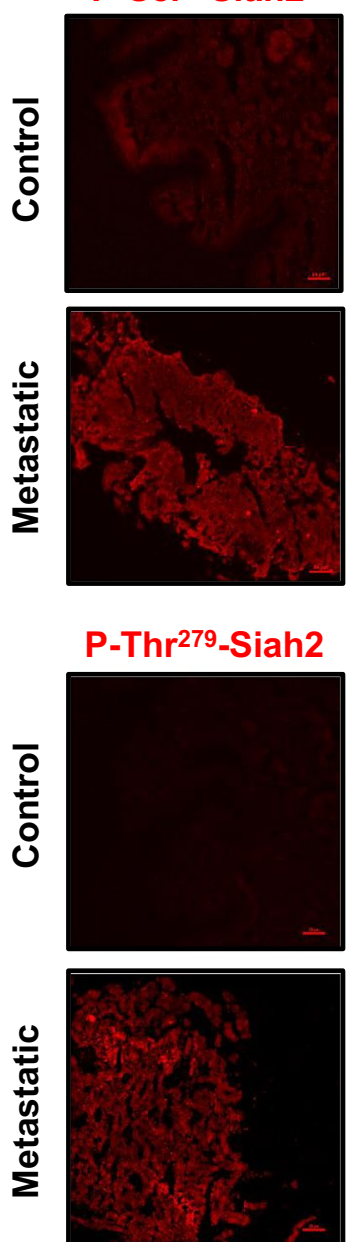

Siah2
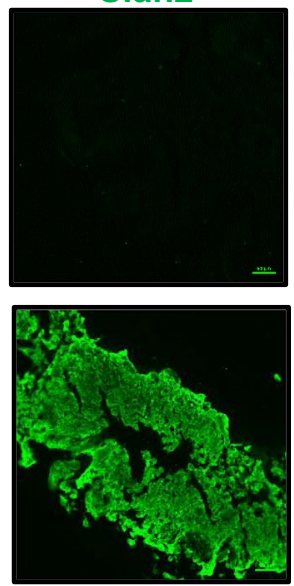

Siah2
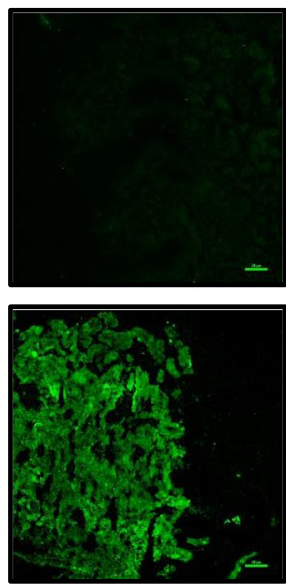

DAPI
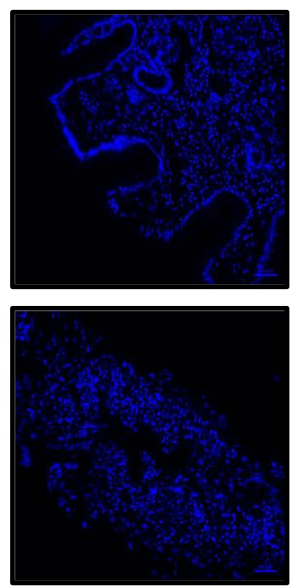

DAPI
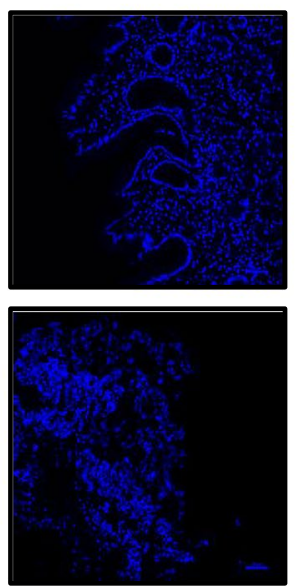

Merged
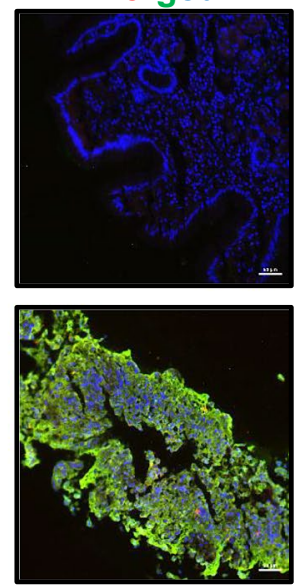

Merged
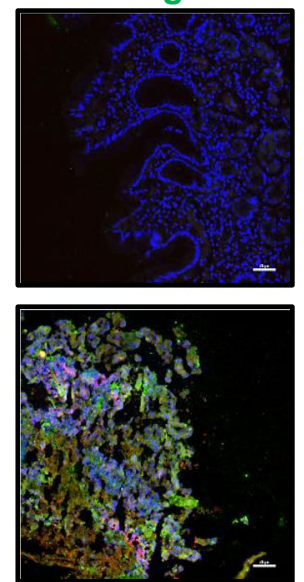

Fig. 5 Increase in P-Ser/Thr-Siah2 in GECs and in human GC samples. a A representative western blot $(n=3)$ of uninfected and infected (200 MOI H. pylori, 12 h) MKN45 and AGS cells showing enhanced P-Ser ${ }^{6}$-Siah2, P-Thr ${ }^{279}$-Siah2 and Siah2 proteins. GAPDH is the loading control. $\mathbf{b}$ Representative western blot $(n=3)$ of uninfected and infected (200 MOI H. pylori, 12 h) HFE145 cells showing enhanced P-Ser ${ }^{6}$-Siah2, P-Thr ${ }^{279}$-Siah2 and Siah2 proteins. GAPDH is the loading control. $\mathbf{c}$ Immunofluorescence microscopy images of human gastric biopsy tissue $(n=9)$ showing the status of Siah2, P-Ser ${ }^{6}-$ Siah2 (above) and P-Thr ${ }^{279}$-Siah2 (below) proteins. Tissues are sectioned at $5 \mu \mathrm{m}$ thickness. Images are captured using $20 \times$ objective and scale bars represent $50 \mu \mathrm{m}$ 
(See figure on next page.)

Fig. 6 H. felis-infected C57BL/6 mice exhibit enhanced Siah2 phosphorylation and MRCK $\beta$ downregulation. a Immunofluorescence microscopy images of antral gastric tissues of uninfected and infected mice showing the status of Siah2 and MRCK $\beta$. $\mathbf{b}$ Immunofluorescence microscopy images of antral gastric tissues of C57BL/6 mice showing the status of MRCKR, P-Ser ${ }^{6}-$ Siah2 and P-Thr ${ }^{279}$-Siah2. All tissues are sectioned at $5 \mu \mathrm{m}$ thickness. All images are captured using $10 \times$ objective and scale bars represent $100 \mu \mathrm{m}$

performed using $m r c k \beta$-overexpressed or empty vectoroverexpressed AGS cells followed by $12 \mathrm{~h}$ of $\mathrm{H}$. pylori infection. Reaffirming our previous results, representative data $(\mathrm{n}=3)$ showed enhanced levels of P-Ser ${ }^{6}-\mathrm{Siah} 2$ and P-Thr ${ }^{279}$-Siah2 in H. pylori-infected GEC (Additional file 1: Fig. S4).

\section{H. felis infection degrades MRCK $\beta$ but enhances Siah2 and its phosphorylation in $\mathrm{C} 57 \mathrm{BL} / 6$ mice}

H. felis-infected C57BL/6 mice exhibit GC progression events similar to that of humans $[9,31]$. Murine antral gastric samples were probed for MRCK $\beta$ and Siah2 proteins by immunofluorescence microscopy. MRCK $\beta$ was downregulated and Siah2 was upregulated in infected tissues as compared to uninfected controls (Fig. 6a). Immunofluorescence microscopy of infected murine antral gastric samples also exhibited increased $\mathrm{P}^{\mathrm{S}} \mathrm{Ser}^{6}-\mathrm{Siah} 2$ (above) and P-Thr ${ }^{279}$-Siah2 (below) as compared to uninfected controls (Fig. 6b). Therefore, these in vivo findings further strengthened our in vitro results.

Taken together, data shown in Figs. 2, 3, 4, 5, 6 indicated that Helicobacter-mediated Siah2 phosphorylation at $\operatorname{Ser}^{6}$ and $\mathrm{Thr}^{279}$ was driven by MRCK $\beta$ but Siah2MRCK $\beta$ interaction degraded MRCK $\beta$.

\section{Phosphorylation of Siah2 promotes GC progression and invasiveness}

Siah2 and its acetylation enhances GC invasion and metastasis [7-9]. In order to investigate role of Siah2 phosphorylation on anchorage-independent growth of GEC, pcDNA3.1+, WT and phospho-null mutant siah2expressing MKN45 stable cells were either left uninfected or were infected for $12 \mathrm{~h}$ at $200 \mathrm{MOI}$ and used for soft agar assay. We found a significantly decreased number of colonies in case of Siah2 phospho-null mutants as compared to siah2 WT, which formed maximum number of colonies after $H$. pylori infection (Fig. 7a). Anchoragedependent growth of these stable cells were assessed. Cells were either left uninfected or were challenged with $200 \mathrm{MOI} H$. pylori-for $12 \mathrm{~h}$. We found a significant increase in the number of colonies formed in siah2 WT cells as compared to the empty vector and Siah2 phospho-null mutant-expressing cells (Fig. 7b). Influence of Siah2 phosphorylation on invasion was examined using
pcDNA3.1+, siah2 WT and phospho-null mutant siah2expressing AGS stable cells. MKN45 cells are semi-adherent in nature and, therefore, were not used for invasion assays $[8,9]$. Invasive potential of siah2 WT stable cells was significantly higher as compared to their phosphonull counterparts (Fig. 7c). Role of Siah2 phosphorylation on cellular proliferation was assessed by MTT assay and cell population doubling assay using $H$. pylori-infected or uninfected pcDNA3.1+, siah2 WT and Siah2 phosphonull mutant-expressed MKN45 stable cells. We found that proliferation of Siah2 phospho-null mutant cells was significantly less than siah 2 WT cells $12 \mathrm{~h}$ post-infection (Fig. $7 \mathrm{~d}$ and e). Altogether, these results establish that Siah2 phosphorylation had an important role in GC progression and invasiveness.

\section{Discussion}

Siah2 is upregulated in response to $H$. pylori infection and promotes proliferation as well as invasiveness of GECs $[7,8]$. The main finding of this study is that Siah2 is phosphorylated by the Ser/Thr Kinase MRCK $\beta$ in GECs infected with $H$. pylori. Phosphorylation enhances Siah2 stability and our results reveal the importance of Siah2 phosphorylation in increasing GEC survival, carcinogenic/proliferative potential, invasiveness and anchorageindependent growth. Therefore, interference of Siah2 phosphorylation might be a useful therapeutic approach for the treatment of GC.

Dynamic phosphorylation-dephosphorylation events modulate many cellular processes. Phosphorylation of proteins often act as on-off switches for protein function. Apoptosis-regulatory proteins and proteins involved in EMT are very tightly regulated by differential phosphorylation [32-34]. Phosphorylation controls enzyme activities and phosphorylation-mediated deregulations of ubiquitinating enzymes are major threats in causing progression of cancer and degenerative diseases [35]. Phosphorylation of the E3 ubiquitin ligase Siah2 is pivotal in regulating its function [11-14]. This study has identified for the first time that Siah2 is phosphorylated at $\operatorname{Ser}^{6}$ and $\mathrm{Th}^{279}$ residues by MRCK $\beta$ in H. pylori-infected GECs, human antral GC biopsy samples and $H$. felis-infected murine gastric tissues. Our results are supported by a mass spectrometry-based phosphoproteome analysis of a myelogenous leukemia cell line K562 which also identified the presence of $\mathrm{Ser}^{6}$-phosphorylated Siah2 in that 

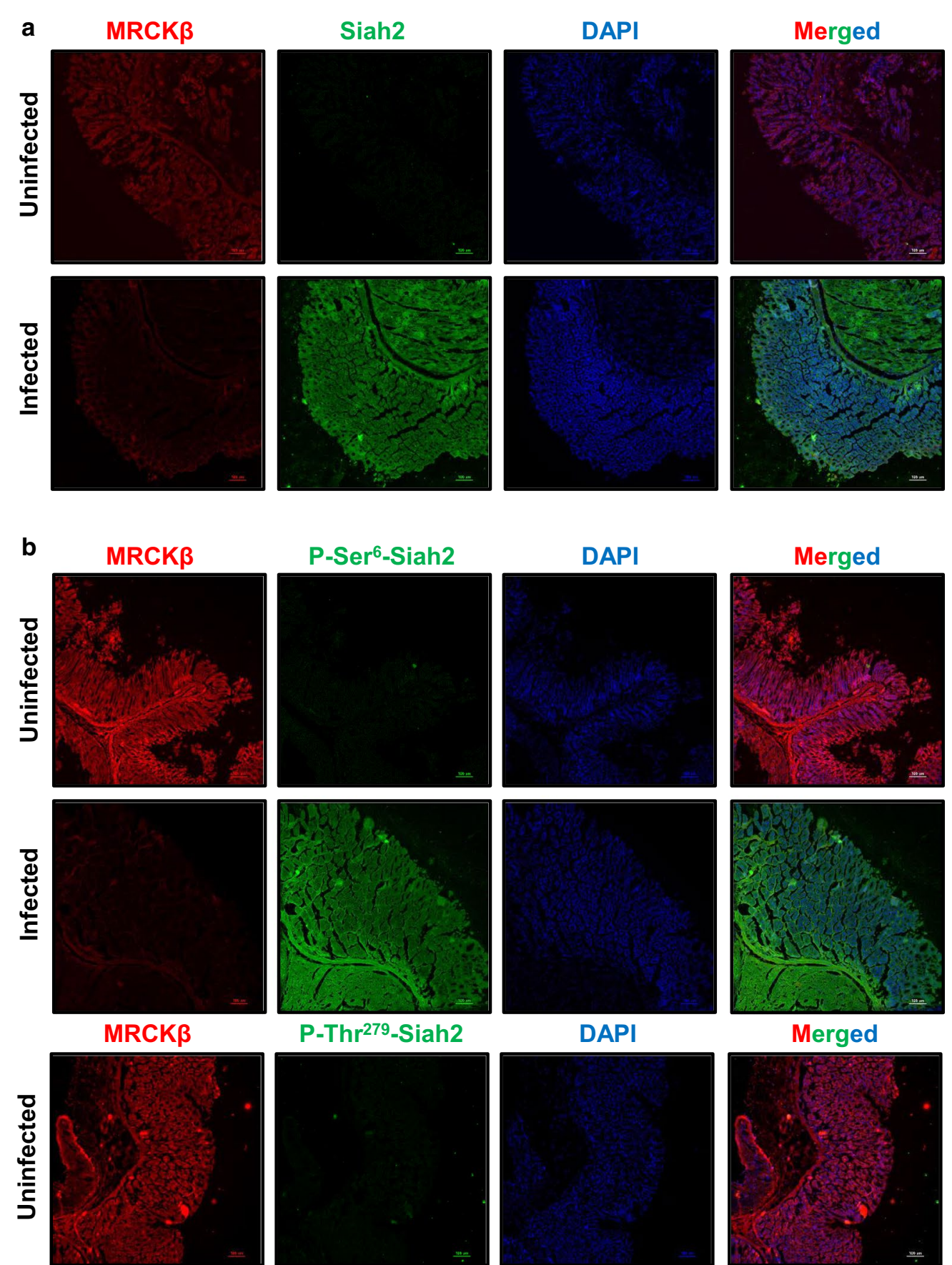

$$
\text { P-Thr279-Siah2 }
$$

DAPI

Merged
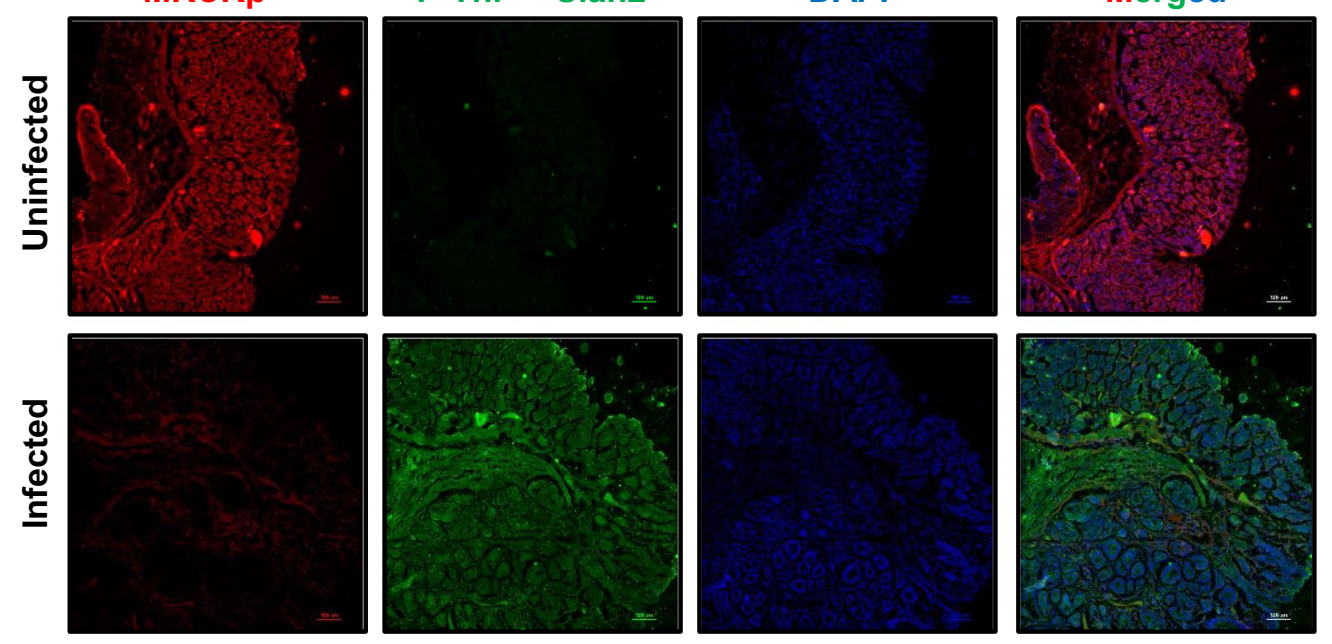


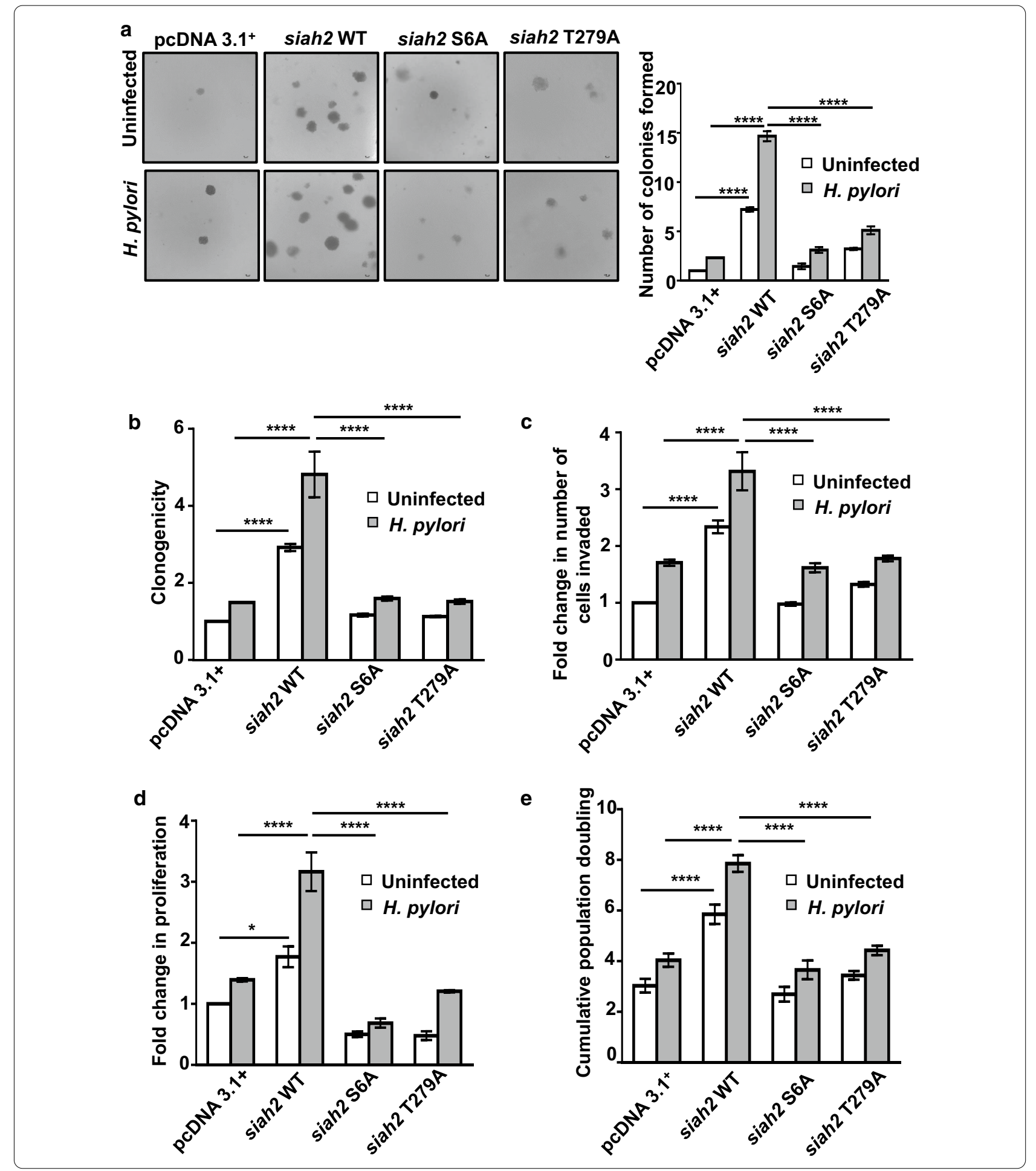

cell but identifying the functional importance of phospho-Siah2 was not within the scope of that study [30]. Along with phosphorylation at $\operatorname{Ser}^{6}$ we have identified phosphorylation of Siah2 at $\mathrm{Thr}^{279}$ and elucidated their functional importance in GC progression. The inability to undergo phosphorylation at $\operatorname{Ser}^{6}$ and $\mathrm{Thr}^{279}$ decreases the tumorigenic functions of Siah2. Future cross-cancer studies would help us determine whether Siah2 phosphorylation is essential for carcinogenic processes. 
(See figure on previous page.)

Fig. 7 Phosphorylated Siah2 regulates GC progression. a Brightfield images showing colonies formed in soft agar assay $(n=3)$ by pcDNA3.1+, WT siah2, S6A and T279A siah2 mutant-expressing MKN45 stable cells. Images are taken using 4X objective and scale bar represents $100 \mu \mathrm{m}$. Colonies greater than $50 \mu \mathrm{m}$ are counted. b Bar graph represents change in the colony formation ability of pcDNA3.1+, WT siah2 and Siah2 phospho-null mutant stably-expressing MKN45 cells. Results are normalized to the uninfected empty vector control. c Graphical representation of changes in the invasive potential of pcDNA3.1+, siah2 WT and Siah2 phospho-null mutant-expressing AGS stable cells as assessed by the number of invaded cells. Values are normalized to uninfected empty vector control. $\mathbf{d}$ Graphical representation of change in cellular proliferation assessed by MTT assay using pcDNA3.1+, siah2 WT and Siah2 phospho-null mutant-expressing MKN45 stable cells. e Graphical representation of cumulative population doubling of uninfected and H. pylori-infected pcDNA3.1+, siah2 WT and Siah2 phospho-null mutant-expressing MKN45 stable cells. In all of these panels two-way ANOVA following Tukey's post hoc analysis calculates the statistical significance. All data represent mean $\pm \operatorname{sem}(n=3), * P<0.05$, $* * * * P<0.0001$

MRCK $\beta$ belongs to a subfamily of Rho GTPases within the AGC family of kinases (i.e., protein kinases A, G and C). MRCK $\beta$ interacts with $\mathrm{Cdc} 42$ and regulates myosin II-regulatory light chain phosphorylation important for the regulation of actin-myosin cytoskeleton dynamics $[18,36-38]$. By studies involving overexpression we show that MRCK $\beta$ is responsible for Siah2 phosphorylation. Our observation shows that although MRCK $\beta$ is a kinase phosphorylating Siah2 in $H$. pylori-challenged GECs, Siah2 is a "kiss of death" signal for MRCK $\beta$. We find that Siah2 ubiquitinates MRCK $\beta$. MRCK $\beta$ ubiquitination has been reported by a multiplexed mass spectrometry study in various cells and tissue samples including cancer [39]. This study is also in harmony with another observation which identified that Siah2 could regulate the cellular pool of its kinases through ubiquitination [40]. Most of the ubiquitinated proteins are targeted for proteasomal degradation [41, 42] and our results also point towards the proteasomal degradation of MRCK $\beta$ in $H$. pylori-infected GECs. Although there is no report on the status and role of MRCK $\beta$ in GC, our finding is supported by the human protein atlas information on "stomach cancer" showing that only $16.67 \%$ GC samples moderately express Cdc42BPB (MRCK $\beta)$ and the rest do not stain for the protein (v19.proteinatlas. org/ENSG00000198752-CDC42BPB/pathology) while $80 \%$ of GC tissues show expression of Siah2 protein (v19. proteinatlas.org/ENSG00000181788-SIAH2/pathology) [25]. Cdc42 is a small GTPase which is implicated in the progression of cancer via regulation of many cellular processes [31]. Cdc42 regulates tumorigenesis by modulating several kinases that are known for their tumorigenic potential $[21,41]$. Since GC cell motility and polarity are highly dependent on the Rho GTPase Cdc42 [43, 44], it is imperative to study how the loss of Cdc42BPB i.e. MRCK $\beta$ is compensated in GC.

\section{Conclusions}

In conclusion, our study shows that MRCK $\beta$-mediated phosphorylation of Siah2 increases proliferation, survival, anchorage-independent nature and invasiveness of GECs implicating a crucial link of the mechanism with GC progression. In addition, this study establishes that phosphorylation-mediated increased stability of Siah2 and concomitant decrease in MRCK $\beta$ are important immunohistochemical characteristics of metastatic GC. As Siah2 stabilization by H. pylori ensures GC progression, targeting Siah2 stability looks very promising for therapeutic intervention of GC.

\section{Supplementary Information}

The online version contains supplementary material available at https://doi. org/10.1186/s12929-021-00710-0.

Additional file 1: Fig. S1. Result of LR_PPI analysis showing the probability of Siah2-MRCK $\beta$ interaction. Fig. S2. Identification of novel phosphorylatable residues. (A) Novel Siah2 phosphorylatable residues along with their predicted scores as identified using NetPhos 3.0. (B) Sequence and multi-domain schematic depiction of human Siah2 protein. Identified highly probable phosphorylatable amino acids are underlined in the sequence and represented by vertical lines in the domain structure. Fig. S3i. Human gastric biopsy tissues exhibit enhanced Siah2 phosphorylation and MRCK $\beta$ downregulation. Immunofluorescence microscopy images showing the status of Siah2, P-Ser ${ }^{6}-S_{i a h 2,}$ P-Thr ${ }^{279}-\operatorname{Siah} 2$ and MRCK $\beta$ in Patient \#1-3. Objective: 20X and scale bar represents $50 \mu \mathrm{m}$. Fig. S3ii. Human gastric biopsy tissues exhibit enhanced Siah2 phosphorylation and MRCK $\beta$ downregulation. Immunofluorescence microscopy images showing the status of Siah2, P-Ser ${ }^{6}-S_{i a h 2}, \mathrm{P}-\mathrm{Thr}^{279}-\mathrm{Siah} 2$ and MRCK $\beta$ in Patient \#4-6. Objective: 20X and scale bar represents $50 \mu \mathrm{m}$. Fig. S3iii. Human gastric biopsy tissues exhibit enhanced Siah2 phosphorylation and MRCK $\beta$ downregulation. Immunofluorescence microscopy images showing the status of Siah2, P-Ser ${ }^{6}-S_{i a h 2, ~ P-T h r^{279}-S i a h 2}$ and MRCK $\beta$ in Patient \#7-9. Objective: 20X and scale bar represents $50 \mu \mathrm{m}$. Fig. S4. Phosphorylated Siah 2 is enhanced upon mrck $\beta$ overexpression. Immunofluorescence microscopy images of AGS cells overexpressing the empty vector or mrck $\beta$ and infected with $200 \mathrm{MOl} H$. pylori for $12 \mathrm{~h}$ showing enhanced P-Ser ${ }^{6}$-Siah2 and P-Thr ${ }^{279}$-Siah2. Objective: 60X and scale bar represents $20 \mu \mathrm{m}$.

\section{Abbreviations}

cag PAl: Cytotoxin associated gene pathogenicity island; GC: Gastric cancer; GECs: Gastric epithelial cells; H. pylori: Helicobacter pylori; MG132: Z-Leu-LeuLeu-al; MOI: Multiplicity of infection; MRCKß: Myotonic dystrophy kinaserelated Cdc42-binding kinase $\beta$; P-Ser ${ }^{6}$-Siah2: Phosphorylated Serine ${ }^{6}$ Siah2; P-Thr ${ }^{279}$-Siah2: Phosphorylated Threonine ${ }^{6}$ Siah2; Siah: Seven in absentia homolog; WT: Wild type.

\section{Acknowledgements}

P.D., I.P. and D.C. obtained fellowships from DAE, India. Central Instrumentation Facilities of NISER and Centre for Interdisciplinary Sciences (CIS), NISER are acknowledged for providing facilities. 


\section{Authors' contributions}

P.D. performed the experiments, analysed the results and wrote the paper; S.B.K. performed site-directed mutagenesis; I.P. and D.C. assisted in manuscript preparation and arranging the figures; H.A. and D.T.S. provided HFE145 cell line; N.R. and S.P.S. helped with gastric cancer biopsy collection and analysis; A.B. conceived the work, designed experiments, supervised the work, analysed the data and wrote the paper. All authors read and approved the final manuscript.

\section{Funding}

This work was supported by the Indian Science and Engineering Research Board grant (SB/SO/BB-0015/2014) (to A.B.)

\section{Availability of data and materials}

The datasets used and/or analysed during the current study are available from the corresponding author on reasonable request.

\section{Ethics approval and consent to participate}

Human GC biopsy tissue collection was approved by Institutional Ethics Committee for Human Research, National Institute of Science Education and Research (NISER) (protocol No. NISER/HEC/2012/AB-1) and a prior written consent of the patients was obtained. For experiments involving use of animals, approval of the Institutional Animal Ethics Committee of NISER was obtained (protocol No. NISER/SBS/IAEC/AH-34).

\section{Consent for publication}

Not applicable.

\section{Competing interests}

The authors declare no competing or financial interests.

\begin{abstract}
Author details
${ }^{1}$ School of Biological Sciences, National Institute of Science Education and Research (NISER) Bhubaneswar, HBNI, P.O. Bhimpur-Padanpur, Via Jatni, Khurda 752050, Odisha, India. ${ }^{2}$ Department of Medicine, Meharry Medical Center, Nashville, TN 37208, USA. ${ }^{3}$ Department of Medicine, Howard University, Washington, DC 20060, USA. ${ }^{4}$ Department of Pathology, Acharya Harihar Post Graduate Institute of Cancer, Cuttack 753007, Odisha, India. ${ }^{5}$ Department of Gastroenterology, SCB Medical College, Cuttack 753007, Odisha, India.

${ }^{6}$ Present Address: Institute of Biotechnology, University of Helsinki, P.O. Box 56 , 0014 Helsinki, Finland.
\end{abstract}

Received: 9 November 2020 Accepted: 22 January 2021

Published online: 03 February 2021

\section{References}

1. Wang F, Meng W, Wang B, Qiao L. Helicobacter pylori-induced gastric inflammation and gastric cancer. Cancer Lett. 2014;345(2):196-202.

2. Zhang $X-Y$, Zhang P-Y, Aboul-Soud MA. From inflammation to gastric cancer: role of Helicobacter pylori. Oncology letters. 2017;13(2):543-8.

3. Wroblewski LE, Peek RM. Helicobacter pylori, cancer, and the gastric microbiota. In: Cells S, editor. Pre-neoplasia, and early cancer of the upper gastrointestinal tract. Springer: Cham; 2016. p. 393-408.

4. Waskito LA, Salama NR, Yamaoka Y. Pathogenesis of Helicobacter pylori infection. Helicobacter. 2018;23:e12516.

5. Krämer OH, Stauber RH, Bug G, Hartkamp J, Knauer SK. SIAH proteins: critical roles in leukemogenesis. Leukemia. 2013;27(4):792-802.

6. Adam MG, Matt S, Christian S, Hess-Stumpp H, Haegebarth A, Hofmann TG, et al. SIAH ubiquitin ligases regulate breast cancer cell migration and invasion independent of the oxygen status. Cell Cycle. 2015;14(23):3734-47.

7. Kokate SB, Dixit P, Das L, Rath S, Roy AD, Poirah I, et al. Acetylationmediated Siah2 stabilization enhances PHD3 degradation in Helicobacter pylori-infected gastric epithelial cancer cells. FASEB J. 2018;32(10):5378-89.

8. Das L, Kokate SB, Rath S, Rout N, Singh SP, Crowe SE, et al. ETS2 and Twist1 promote invasiveness of Helicobacter pylori-infected gastric cancer cells by inducing Siah2. Biochem J. 2016;473(11):1629-40.
9. Kokate SB, Dixit P, Poirah I, Roy AD, Chakraborty D, Rout N, et al. Testin and filamin-C downregulation by acetylated Siah2 increases invasiveness of Helicobacter pylori-infected gastric cancer cells. Int J Biochem Cell Biol. 2018;103:14-24.

10. Khurana A, Nakayama K, Williams S, Davis RJ, Mustelin T, Ronai Z. Regulation of the ring finger E3 ligase Siah2 by p38 MAPK. J Biol Chem. 2006;281(46):35316-26.

11. Pérez M, García-Limones C, Zapico I, Marina A, Schmitz ML, Muñoz E, et al. Mutual regulation between SIAH2 and DYRK2 controls hypoxic and genotoxic signaling pathways. J Mol Cell Biol. 2012;4(5):316-30.

12. Sarkar TR, Sharan S, Wang J, Pawar SA, Cantwell CA, Johnson PF, et al. Identification of a Src tyrosine kinase/SIAH2 E3 ubiquitin ligase pathway that regulates C/EBPS expression and contributes to transformation of breast tumor cells. Mol Cell Biol. 2012;32(2):320-32.

13. Grishina I, Debus K, García-Limones C, Schneider C, Shresta A, García C, et al. SIAH-mediated ubiquitination and degradation of acetyl-transferases regulate the p53 response and protein acetylation. Biochimica et Biophysica Acta (BBA) Mol Cell Res. 2012;1823(12):2287-96.

14. Garcia-Limones C, Lara-Chica M, Jimenez-Jimenez C, Perez M, Moreno $P$, Munoz E, et al. CHK2 stability is regulated by the E3 ubiquitin ligase SIAH2. Oncogene. 2016;35(33):4289-301.

15. Huo L, Wen W, Wang R, Kam C, Xia J, Feng W, et al. Cdc42-dependent formation of the ZO-1/MRCK $\beta$ complex at the leading edge controls cell migration. EMBO J. 2011;30(4):665-78.

16. Gaggioli C, Hooper S, Hidalgo-Carcedo C, Grosse R, Marshall JF, Harrington $\mathrm{K}$, et al. Fibroblast-led collective invasion of carcinoma cells with differing roles for RhoGTPases in leading and following cells. Nat Cell Biol. 2007;9(12):1392-400.

17. Rafn B, Nielsen CF, Andersen SH, Szyniarowski P, Corcelle-Termeau E,

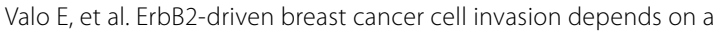
complex signaling network activating myeloid zinc finger-1-dependent cathepsin B expression. Mol Cell. 2012;45(6):764-76.

18. Unbekandt M, Belshaw S, Bower J, Clarke M, Cordes J, Crighton D, et al. Discovery of potent and selective MRCK inhibitors with therapeutic effect on skin cancer. Cancer Res. 2018;78(8):2096-114.

19. Talman V, Gateva G, Ahti M, Ekokoski E, Lappalainen P, Tuominen RK. Evidence for a role of MRCK in mediating HeLa cell elongation induced by the C1 domain ligand HMI-1a3. Eur J Pharm Sci. 2014;55:46-57.

20. Das L, Kokate S, Dixit P, Rath S, Rout N, Singh S, et al. Membrane-bound $\beta$-catenin degradation is enhanced by ETS2-mediated Siah1 induction in Helicobacter pylori-infected gastric cancer cells. Oncogenesis. 2017;6(5):e327-e.

21. Zhu Z, Yu Z, Rong Z, Luo Z, Zhang J, Qiu Z, et al. The novel GINS4 axis promotes gastric cancer growth and progression by activating Rac1 and CDC42. Theranostics. 2019;9(26):8294.

22. Du DS, Yang XZ, Wang Q, Dai WJ, Kuai WX, Liu YL, et al. Effects of CDC42 on the proliferation and invasion of gastric cancer cells. Mol Med Rep. 2016;13(1):550-4.

23. Bruder SP, Jaiswal N, Haynesworth SE. Growth kinetics, self-renewal, and the osteogenic potential of purified human mesenchymal stem cells during extensive subcultivation and following cryopreservation. J Cell Biochem. 1997;64(2):278-94.

24. Greenwood SK, Hill RB, Sun JT, Armstrong MJ, Johnson TE, Gara JP, et al. Population doubling: a simple and more accurate estimation of cell growth suppression in the in vitro assay for chromosomal aberrations that reduces irrelevant positive results. Environ Mol Mutagen. 2004;43(1):36-44

25. Petrenko Y, Vackova I, Kekulova K, Chudickova M, Koci Z, Turnovcova K, et al. A comparative analysis of multipotent mesenchymal stromal cells derived from different sources, with a focus on neuroregenerative potential. Sci Rep. 2020;10(1):1-15.

26. Pan $X-Y$, Zhang $Y-N$, Shen H-B. Large-Scale prediction of human proteinprotein interactions from amino acid sequence based on latent topic features. J Proteome Res. 2010;9(10):4992-5001.

27. Ma B, Cheng H, Mu C, Geng G, Zhao T, Luo Q, et al. The SIAH2-NRF1 axis spatially regulates tumor microenvironment remodeling for tumor progression. Nat Commun. 2019;10(1):1-17.

28. Zhang Y, Zheng X, Tan H, Lu Y, Tao D, Liu Y, et al. PIWIL2 suppresses Siah2mediated degradation of HDAC3 and facilitates CK2a-mediated HDAC3 phosphorylation. Cell Death Dis. 2018;9(4):1-11. 
29. Blom N, Gammeltoft S, Brunak S. Sequence and structure-based prediction of eukaryotic protein phosphorylation sites. J Mol Biol. 1999;294(5):1351-62.

30. Zhou H, Di Palma S, Preisinger C, Peng M, Polat AN, Heck AJ, et al. Toward a comprehensive characterization of a human cancer cell phosphoproteome. J Proteome Res. 2013;12(1):260-71.

31. del Mar MM, Dharmawardhane S. Targeting rac and Cdc42 GTPases in cancer. Cancer Res. 2018;78(12):3101-11.

32. Rath S, Das L, Kokate SB, Pratheek B, Chattopadhyay S, Goswami C, et al Regulation of Noxa-mediated apoptosis in Helicobacter pylori-infected gastric epithelial cells. FASEB J. 2015;29(3):796-806.

33. Ashcroft M, Kubbutat MH, Vousden $\mathrm{KH}$. Regulation of $\mathrm{p} 53$ function and stability by phosphorylation. Mol Cell Biol. 1999;19(3):1751-8.

34. Tang H, Massi D, Hemmings BA, Mandalà M, Hu Z, Wicki A, et al. AKT-ions with a TWIST between EMT and MET. Oncotarget. 2016;7(38):62767.

35. Barber KW, Rinehart J. The abcs of ptms. Nat Chem Biol. 2018;14(3):188-92.

36. Unbekandt M, Olson MF. The actin-myosin regulatory MRCK kinases: regulation, biological functions and associations with human cancer. J Mol Med. 2014;92(3):217-25.

37. Tan I, Yong J, Dong JM, Lim L, Leung T. A tripartite complex containing MRCK modulates lamellar actomyosin retrograde flow. Cell. 2008;135(1):123-36

38. Pichaud F, Walther RF, de Almeida FN. Regulation of Cdc42 and its effectors in epithelial morphogenesis. J Cell Sci. 2019. https://doi.org/10.1242/ jcs.217869.
39. Rose CM, Isasa M, Ordureau A, Prado MA, Beausoleil SA, Jedrychowski MP, et al. Highly multiplexed quantitative mass spectrometry analysis of ubiquitylomes. Cell Syst. 2016;3(4):395-403.e4.

40. Calzado MA, De La Vega L, Möller A, Bowtell DD, Schmitz ML. An inducible autoregulatory loop between HIPK2 and Siah2 at the apex of the hypoxic response. Nat Cell Biol. 2009;11(1):85-91.

41. Parrini MC, Camonis J, Matsuda M, de Gunzburg J. Dissecting activation of the PAK1 kinase at protrusions in living cells. J Biol Chem. 2009;284(36):24133-43.

42. Hoeller D, Dikic I. How the proteasome is degraded. Proc Natl Acad Sci. 2016;113(47):13266-8.

43. Li $X$, Jiang $M$, Chen D, Xu B, Wang R, Chu Y, et al. miR-148b-3p inhibits gastric cancer metastasis by inhibiting the Dock6/Rac1/Cdc42 axis. J Exp Clin Cancer Res. 2018;37(1):71.

44. Osman MA, Bloom GS, Tagoe EA. Helicobacter pylori-induced alteration of epithelial cell signaling and polarity: a possible mechanism of gastric carcinoma etiology and disparity. Cytoskeleton. 2013;70(7):349-59.

\section{Publisher's Note}

Springer Nature remains neutral with regard to jurisdictional claims in published maps and institutional affiliations.
Ready to submit your research? Choose BMC and benefit from:

- fast, convenient online submission

- thorough peer review by experienced researchers in your field

- rapid publication on acceptance

- support for research data, including large and complex data types

- gold Open Access which fosters wider collaboration and increased citations

- maximum visibility for your research: over $100 \mathrm{M}$ website views per year

At BMC, research is always in progress.

Learn more biomedcentral.com/submissions 